\title{
Spotting Solid Oxide Fuel Cell Degradation Effects by Electron Microscopy
}

\author{
A. Hessler-Wyser ${ }^{1}$, J. A. Schuler ${ }^{1,2}$, Z. Wuillemin ${ }^{2}$, J. Van herle ${ }^{2}$ \\ 1 Interdisciplinary Centre for Electron Microscopy (CIME), Ecole Polytechnique Fédérale \\ de Lausanne (EPFL), CH-1015 Lausanne, Switzerland \\ 2 Laboratory of Industrial Energy Systems (LENI), Ecole Polytechnique Fédérale de \\ Lausanne (EPFL), CH-1015 Lausanne, Switzerland
}

In the present international energy context, the landscape is being redesigned. Decentralised combined heat and power, fuel diversity and increased use of renewable sources will gain in importance. Solid Oxide Fuel Cells (SOFCs) represent an attractive conversion technology and are considered as part of the answer to decentralised generation at small to medium scale with the highest possible electrical efficiency (60\% even for standalone $\mathrm{kW}$-scale systems).

Although very promising, SOFC technology is not yet mature, as SOFC stack performance slowly degrades during operation, which occurs at different levels. Stack degradation is a crucial technological issue prior to SOFC commercialization, and considerable progress has been achieved. Degradation pathways have been identified, amongst them microstructural changes, poisoning effects and formation of low conductive phases. However many questions still remain regarding the mechanisms involved in these degradation processes.

In this work, tested SOFC were planar anode-supported SOFCs based on nickel and yttriastabilized zirconia (YSZ) composite. The anode was co-sintered with a few micron thick membrane of dense YSZ electrolyte, on top of which a 20-40 $\mu \mathrm{m}$ layer of lanthanum strontium manganite (LSM) and YSZ composite cathode was subsequently deposited. The interconnects used to stack the ceramic cells in series were made of Cr-containing ferritic steels protected by a coating of $\mathrm{MnCo}_{2} \mathrm{O}_{4}$. The test consisted in a segmented repeat element (i.e. a 1-cell stack) operated at $800^{\circ} \mathrm{C}$ for $1900 \mathrm{~h}$. Each segment of the cell could be monitored separately, giving spatially resolved information on the cell performance [1].

On the cathode side, pollution and secondary phase formation alter the cell performances. In particular, pollutant species contained in the air flow can deposit at the cathode/electrolyte interface or at the current collection layer surface and reduce either the catalytic activity or mass transfer e.g. by pore blocking. Cr, even in small quantities, is known to be a severe pollutant, nevertheless its detection by energy dispersive spectroscopy (EDS) along with LSM is challenging due to strong overlaps of the X-ray emission lines of $\mathrm{La}, \mathrm{Mn}$ and $\mathrm{Cr}$.

Multiscale analysis was necessary to localize and quantify the $\mathrm{Cr}$ in the cathode and in particular in the active region at the electrolyte/cathode interface. Combination of optical emission spectroscopy, systematic scanning electron microscopy (SEM) + EDS and spectral simulations demonstrated it was possible to quantify small amounts of $\mathrm{Cr}$ deposited in cathodes at a medium scale [2]. To localize $\mathrm{Cr}$ at smaller scale, transmission electron microscopy (TEM) lamellae were extracted by focused ion beam (FIB) from different segments of the segmented tested cell. The analyses were performed on an FEI Osiris equipped with a X-FEG high brightness electron gun and optimized ChemiSTEM ${ }^{\mathrm{TM}}$ technology. 
Secondary phase formation at the cathode/electrolyte interface was highlighted by STEM/EDS. In particular, strontium zirconate (SZO) formation was evidenced at the interfaces between LSM and YSZ, together with delamination of sintered grains. Although predicted by some authors, SZO observation had not been reported before; our work demonstrated that its presence produces delamination at the SZO/YSZ interface [3]. In addition, the new $\mathrm{X}$-ray detection technology allowed us to highlight the systematic presence of a nanometric $\mathrm{Cr}_{2} \mathrm{O}_{3}$ layer at the YSZ/SZO interface in the active region (fig.1). Surprisingly, it seems not to be linked to triple phase boundaries, suggesting diffusion of $\mathrm{Cr}$ along interfaces. In addition, it appeared to be located at $\mathrm{Mn}$ free interfaces, although in literature the deposition mechanisms are suspected to be $\mathrm{Mn}^{2+}$ driven. These $\mathrm{Cr}$ oxide layers could be observed only close to the electrolyte/cathode interface, in the active region. Likewise, they were not observed in unpolarised segments.

Furthermore, Mn and La diffusion could be evidenced at YSZ grain boundaries in the electrolyte, despite the presence of SZO and Cr oxide layers between LSM and YSZ grains. This effect could also be seen in the YSZ grains of the cathode, but was less pronounced.

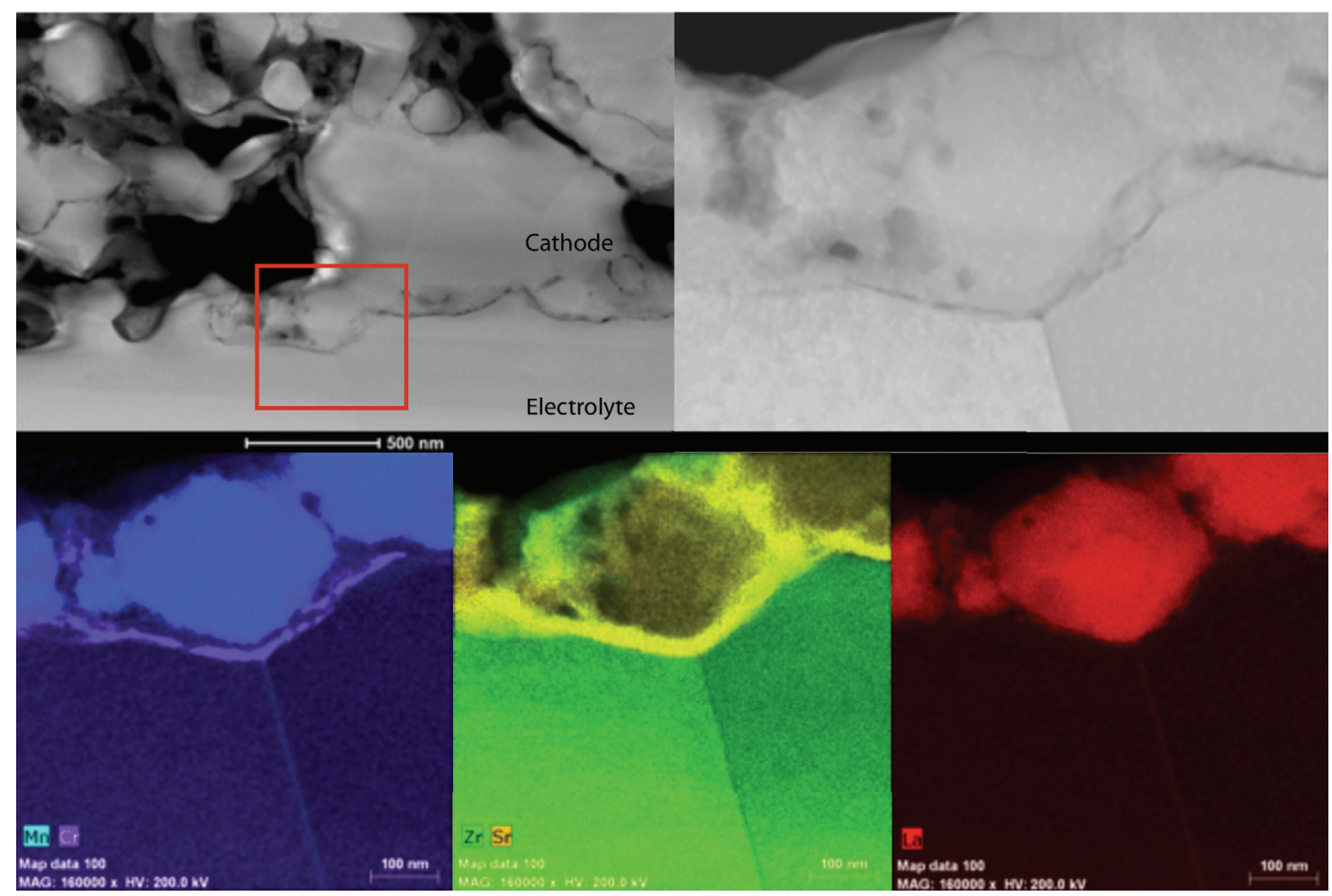

Figure 1. TOP: STEM HAADF image of electrolyte/cathode interface (left) and region of analysis (right), bottom: EDX mapping for Cr-Mn (left), $\mathrm{Zr}$-Sr (middle) and La (right) of the corresponding region.

[1] Wuillemin Z, Nakajo A, Müller A, Schuler AJ, Diethelm S, Van Herle J, Favrat D. ECS Trans 25, (2009). p.457.

[2] Schuler JA, Tanasini P, Hessler-Wyser A, Van herle J. Scripta Materialia (2010) 63:895.

[3] Hessler-Wyser A, Wuillemin Z, Schuler JA, Faes A, Van herle J. J Mater Sci (2011) 46:4532. 